\title{
Curl of Vector Field as Pseudovector
}

\section{Peng $\mathrm{Li}^{1,2}$ a , Xiao-Ping Qin ${ }^{1,2}$, Xue-Ping Cheng ${ }^{1,2}$ and Sheng-Wen $\mathrm{Qi}^{1,2}$}

${ }^{1}$ School of Mathematics, Physics and Information Science, Zhejiang Ocean University, Zhoushan 316022, China

${ }^{2}$ Key Laboratory of Oceanographic Big Data Mining \& Application of Zhejiang Province, Zhoushan 316022, China

apengli.physics@qq.com

Keywords: Pseudovector, Curl, Vector Field.

Abstract. In this paper we investigate another type of pseudovectors: the curl of vector field. The definition of curl is introduced and its transformation property under space rotation and inversion is thoroughly investigated.

\section{Introduction}

In previous papers [1] we have shown that the torque, the angular momentum and the magnetic dipole moment are all pseudovectors defined by the cross product of real vectors. In this paper we further investigate another frequently encountered type of pseudovectors: the curl of vector field.

\section{Definition}

In physics, the terminology field refers to any physical quantity that spread (or distribute) in space. For example, the electrical potential $\varphi(\boldsymbol{r})$, the mass density $\rho(\boldsymbol{r})$, the temperature distribution $T(\boldsymbol{r})$, the air pressure $P(\boldsymbol{r})$, etc., are all fields of scalar quantities. There are fields of vectors too, for example, the electric field intensity $\boldsymbol{E}(\boldsymbol{r})$, the velocity of a fluid $\boldsymbol{v}(\boldsymbol{r})$, the gravitational force $\boldsymbol{G}(\boldsymbol{r})$, and the magnetic field $\boldsymbol{B}(\boldsymbol{r})$, etc. Therefore we have both scalar fields and vector fields.

For vector fields, it is useful to describe their mathematical structure via local differentials. Generally speaking, for a 3D vector field $\boldsymbol{A}(\boldsymbol{r})$, there are totally nine available partial differential functions :

$$
\frac{\partial A_{x}}{\partial x}, \frac{\partial A_{y}}{\partial x}, \frac{\partial A_{z}}{\partial x}, \frac{\partial A_{x}}{\partial y}, \frac{\partial A_{y}}{\partial y}, \frac{\partial A_{z}}{\partial y}, \frac{\partial A_{x}}{\partial z}, \frac{\partial A_{y}}{\partial z}, \frac{\partial A_{z}}{\partial z}
$$

It would be very cumbersome to investigate these differential functions one by one. Fortunately, only two linear combinations of these differentials are important in vector analysis theory, the divergence and the curl. The divergence is not the interest of this paper and we focus the curl below.

The curl of a vector field $\boldsymbol{A}(\boldsymbol{r})$ is defined by the following determinant [2]

$$
\nabla \times \boldsymbol{A}=\left|\begin{array}{ccc}
\boldsymbol{e}_{x} & \boldsymbol{e}_{y} & \boldsymbol{e}_{z} \\
\frac{\partial}{\partial x} & \frac{\partial}{\partial x} & \frac{\partial}{\partial x} \\
A_{x} & A_{y} & A_{z}
\end{array}\right| \text {, where } \nabla \equiv\left(\frac{\partial}{\partial x}, \frac{\partial}{\partial y}, \frac{\partial}{\partial z}\right)
$$

Remind that a vector must abide proper transformation rules under rotation, it is crucial to prove that the curl abides these rules to call it a vector. 


\section{Space Rotation}

\subsection{Rotation of Gradient.}

Apparently, if the operator $\nabla$ itself is a vector, then the curl as a 'cross product' of the vector $\nabla$ and the vector field $\boldsymbol{A}$ does look like a vector. Since an operator is not a real quantity, we cannot say directly whether it is a vector or not. Instead we can apply $\nabla$ onto a scalar field $\varphi(\boldsymbol{r})$ and show that $\nabla \varphi(\boldsymbol{r})$ indeed produces a vector field.

We tentatively choose a rotation about the $z$ axis and have the following transformation rule [2]

$$
\boldsymbol{r}=\left(\begin{array}{l}
x \\
y \\
z
\end{array}\right) \stackrel{\boldsymbol{R}_{z, \theta}}{\longrightarrow} \boldsymbol{r}^{\prime}=\left(\begin{array}{l}
x^{\prime} \\
y^{\prime} \\
z^{\prime}
\end{array}\right)=\left(\begin{array}{ccc}
\cos \theta & -\sin \theta & 0 \\
\sin & \cos \theta & 0 \\
0 & 0 & 1
\end{array}\right)\left(\begin{array}{l}
x \\
y \\
z
\end{array}\right)=\left(\begin{array}{c}
x \cos \theta-y \sin \theta \\
x \sin \theta+y \cos \theta \\
z
\end{array}\right) \text {. }
$$

The inverse of the above equation gives

$$
\left(\begin{array}{l}
x \\
y \\
z
\end{array}\right)=\left(\begin{array}{ccc}
\cos \theta & \sin \theta & 0 \\
-\sin & \cos \theta & 0 \\
0 & 0 & 1
\end{array}\right)\left(\begin{array}{l}
x^{\prime} \\
y^{\prime} \\
z^{\prime}
\end{array}\right)=\left(\begin{array}{c}
x^{\prime} \cos \theta+y^{\prime} \sin \theta \\
-x^{\prime} \sin \theta+y^{\prime} \cos \theta \\
z^{\prime}
\end{array}\right) \text {, }
$$

which leads to

$$
\frac{\partial x}{\partial x^{\prime}}=\cos \theta, \frac{\partial y}{\partial x^{\prime}}=-\sin \theta, \frac{\partial x}{\partial y^{\prime}}=\sin \theta, \frac{\partial y}{\partial y^{\prime}}=\cos \theta, \frac{\partial z}{\partial x^{\prime}}=\frac{\partial z}{\partial y^{\prime}}=\frac{\partial x}{\partial z^{\prime}}=\frac{\partial y}{\partial z^{\prime}}=0, \frac{\partial z}{\partial z^{\prime}}=1
$$

Now it is easy to verify that the following chain differentials

$$
\begin{aligned}
& \frac{\partial \varphi}{\partial x^{\prime}}=\frac{\partial \varphi}{\partial x} \frac{\partial x}{\partial x^{\prime}}+\frac{\partial \varphi}{\partial y} \frac{\partial y}{\partial x^{\prime}}+\frac{\partial \varphi}{\partial z} \frac{\partial z}{\partial x^{\prime}}=\frac{\partial \varphi}{\partial x} \cos \theta-\frac{\partial \varphi}{\partial y} \sin \theta \\
& \frac{\partial \varphi}{\partial y^{\prime}}=\frac{\partial \varphi}{\partial x} \frac{\partial x}{\partial y^{\prime}}+\frac{\partial \varphi}{\partial y} \frac{\partial y}{\partial y^{\prime}}+\frac{\partial \varphi}{\partial z} \frac{\partial z}{\partial y^{\prime}}=\frac{\partial \varphi}{\partial x} \sin \theta+\frac{\partial \varphi}{\partial y} \cos \theta \\
& \frac{\partial \varphi}{\partial z^{\prime}}=\frac{\partial \varphi}{\partial x} \frac{\partial x}{\partial z^{\prime}}+\frac{\partial \varphi}{\partial y} \frac{\partial y}{\partial z^{\prime}}+\frac{\partial \varphi}{\partial z} \frac{\partial z}{\partial z^{\prime}}=\frac{\partial \varphi}{\partial z}
\end{aligned}
$$

Recall that the field $\varphi(x, y, z)$ transforms to $\varphi\left(x^{\prime}, y^{\prime}, z^{\prime}\right)$ and the operator $\nabla$ transforms to $\nabla^{\prime}=\left(\partial / \partial x^{\prime}, \partial / \partial y^{\prime}, \partial / \partial z^{\prime}\right)$. Then whether $\nabla \varphi$ is a vector or not depends on the validity of the following equations

$$
\nabla^{\prime} \varphi\left(x^{\prime}, y^{\prime}, z^{\prime}\right)=\boldsymbol{R}_{z, \theta} \nabla \varphi(x, y, z),
$$

i.e.,

$$
\left(\begin{array}{l}
\partial \varphi / \partial x^{\prime} \\
\partial \varphi / \partial y^{\prime} \\
\partial \varphi / \partial z^{\prime}
\end{array}\right)=\left(\begin{array}{ccc}
\cos \theta & -\sin \theta & 0 \\
\sin & \cos \theta & 0 \\
0 & 0 & 1
\end{array}\right)\left(\begin{array}{l}
\partial \varphi / \partial x \\
\partial \varphi / \partial y \\
\partial \varphi / \partial z
\end{array}\right)
$$

It is easy to observe that equation (8) is exactly the same as equation (6). Therefore $\nabla \varphi$ is truly a vector field, which is usually called the gradient of $\varphi(\boldsymbol{r})$. 


\subsection{Rotation of Curl.}

However, because curl is not defined on scalar field, the above procedure is hardly useful. Actually, we have to thoroughly show that the components of $\nabla \times \boldsymbol{A}$ transform properly under arbitrary rotation. Next we show this step by step.

Take the $z$ - rotation as an example, the vector $\boldsymbol{A}$ itself transforms as

$$
\boldsymbol{A}=\left(\begin{array}{c}
A_{x} \\
A_{y} \\
A_{z}
\end{array}\right) \stackrel{\boldsymbol{R}_{z, \theta}}{\longrightarrow} \boldsymbol{A}^{\prime}=\left(\begin{array}{c}
A_{x}{ }^{\prime} \\
A_{y}{ }^{\prime} \\
A_{z}{ }^{\prime}
\end{array}\right)=\left(\begin{array}{ccc}
\cos \theta & -\sin \theta & 0 \\
\sin & \cos \theta & 0 \\
0 & 0 & 1
\end{array}\right)\left(\begin{array}{l}
A_{x} \\
A_{y} \\
A_{z}
\end{array}\right)=\left(\begin{array}{c}
A_{x} \cos \theta-A_{y} \sin \theta \\
A_{x} \sin \theta+A_{y} \cos \theta \\
A_{z}
\end{array}\right) .
$$

Using equation (9) and the chain rules of differentiation we find the $z$-component of curl abides

$$
\begin{aligned}
(\nabla \times \boldsymbol{A})_{z}^{\prime} & =\frac{\partial A_{y}^{\prime}}{\partial x^{\prime}}-\frac{\partial A_{x}{ }^{\prime}}{\partial y^{\prime}}=\left(\frac{\partial A_{x}}{\partial x^{\prime}}+\frac{\partial A_{y}}{\partial y^{\prime}}\right) \sin \theta+\left(\frac{\partial A_{y}}{\partial x^{\prime}}-\frac{\partial A_{x}}{\partial y^{\prime}}\right) \cos \theta \\
& =\left(\left(\frac{\partial A_{x}}{\partial x} \frac{\partial x}{\partial x^{\prime}}+\frac{\partial A_{x}}{\partial y} \frac{\partial y}{\partial x^{\prime}}+\frac{\partial A_{x}}{\partial z} \frac{\partial z}{\partial x^{\prime}}\right)+\left(\frac{\partial A_{y}}{\partial x} \frac{\partial x}{\partial y^{\prime}}+\frac{\partial A_{y}}{\partial y} \frac{\partial y}{\partial y^{\prime}}+\frac{\partial A_{y}}{\partial z} \frac{\partial z}{\partial y^{\prime}}\right)\right) \sin \theta \\
& +\left(\left(\frac{\partial A_{y}}{\partial x} \frac{\partial x}{\partial x^{\prime}}+\frac{\partial A_{y}}{\partial y} \frac{\partial y}{\partial x^{\prime}}+\frac{\partial A_{y}}{\partial z} \frac{\partial z}{\partial x^{\prime}}\right)-\left(\frac{\partial A_{x}}{\partial x} \frac{\partial x}{\partial y^{\prime}}+\frac{\partial A_{x}}{\partial y} \frac{\partial y}{\partial y^{\prime}}+\frac{\partial A_{x}}{\partial z} \frac{\partial z}{\partial y^{\prime}}\right)\right) \cos \theta \\
& =\left(\frac{\partial A_{x}}{\partial x} \cos \theta-\frac{\partial A_{x}}{\partial y} \sin \theta+\frac{\partial A_{y}}{\partial x} \sin \theta+\frac{\partial A_{y}}{\partial y} \cos \theta\right) \sin \theta \\
& +\left(\frac{\partial A_{y}}{\partial x} \cos \theta-\frac{\partial A_{y}}{\partial y} \sin \theta-\frac{\partial A_{x}}{\partial x} \sin \theta-\frac{\partial A_{x}}{\partial y} \cos \theta\right) \cos \theta \\
& =\frac{\partial A_{y}}{\partial x}-\frac{\partial A_{x}}{\partial y}=(\nabla \times \boldsymbol{A})_{z}
\end{aligned}
$$

(10)

Likewise, the $x$-component of the curl abides

$$
\begin{aligned}
(\nabla \times \boldsymbol{A})^{\prime}{ }_{x} & =\frac{\partial A_{z}{ }^{\prime}}{\partial y^{\prime}}-\frac{\partial A_{y}{ }^{\prime}}{\partial z^{\prime}}=\frac{\partial A_{z}}{\partial y^{\prime}}-\left(\frac{\partial A_{x}}{\partial z^{\prime}} \sin \theta+\frac{\partial A_{y}}{\partial z^{\prime}} \cos \theta\right) \\
& =\frac{\partial A_{z}}{\partial x} \frac{\partial x}{\partial y^{\prime}}+\frac{\partial A_{z}}{\partial y} \frac{\partial y}{\partial y^{\prime}}+\frac{\partial A_{z}}{\partial z} \frac{\partial z}{\partial y^{\prime}}-\left(\frac{\partial A_{x}}{\partial x} \frac{\partial x}{\partial z^{\prime}}+\frac{\partial A_{x}}{\partial y} \frac{\partial y}{\partial z^{\prime}}+\frac{\partial A_{x}}{\partial z} \frac{\partial z}{\partial z^{\prime}}\right) \sin \theta \\
& -\left(\frac{\partial A_{y}}{\partial x} \frac{\partial x}{\partial z^{\prime}}+\frac{\partial A_{y}}{\partial y} \frac{\partial y}{\partial z^{\prime}}+\frac{\partial A_{y}}{\partial z} \frac{\partial z}{\partial z^{\prime}}\right) \cos \theta \\
& =\frac{\partial A_{z}}{\partial x} \sin \theta+\frac{\partial A_{z}}{\partial y} \cos \theta-\frac{\partial A_{x}}{\partial z} \sin \theta-\frac{\partial A_{y}}{\partial z} \cos \theta \\
& =\left(\frac{\partial A_{z}}{\partial y}-\frac{\partial A_{y}}{\partial z}\right) \cos \theta-\left(\frac{\partial A_{x}}{\partial z}-\frac{\partial A_{z}}{\partial x}\right) \sin \theta \\
& =(\nabla \times \boldsymbol{A})_{x} \cos \theta-(\nabla \times \boldsymbol{A})_{y} \sin \theta
\end{aligned}
$$

(11)

In exactly the same method, we find the $y$-component of the curl abides

$$
(\nabla \times \boldsymbol{A})^{\prime}{ }_{y}=\frac{\partial A_{x}{ }^{\prime}}{\partial z^{\prime}}-\frac{\partial A_{z}{ }^{\prime}}{\partial x^{\prime}}=(\nabla \times \boldsymbol{A})_{x} \sin \theta+(\nabla \times \boldsymbol{A})_{y} \cos \theta
$$


As shown by equations (10-12), the curl $\nabla \times \boldsymbol{A}$ transforms exact as the position vector under a $z$-axis rotation. It is routine to show that the curl also transforms properly under rotations about both $x$ and $y$ axes. Therefore, the curl of a vector field abides the required rotational transformation and is indeed a physical vector.

\subsection{Space Inversion.}

Consider the space inversion operation that inverts all base vectors, a real vector reverses its sign for every coordinate, i.e.,

$$
A_{x}{ }^{\prime}=-A_{x}, \quad A_{y}{ }^{\prime}=-A_{y}, A_{z}{ }^{\prime}=-A_{z}, \text { and } x^{\prime}=-x, y^{\prime}=-y, \quad z^{\prime}=-z .
$$

This leads to the direct conclusion

$$
\frac{\partial A_{\alpha}{ }^{\prime}}{\partial \beta^{\prime}}=\frac{\partial\left(-A_{\alpha}\right)}{\partial(-\beta)}=\frac{\partial A_{\alpha}}{\partial \beta} \text {, where } \alpha, \beta=x, y, z \text {. }
$$

(14)

As a result the curl does not change sign under space inversion. That is to say, curl is a pseudovector.

\subsection{Picture.}

Intuitively, the curl is often related with vortex, which is a whirl like pattern of the vector field. For example, the velocity field of a typhoon (tropical hurricane at west Pacific Ocean) has a strong curl at its eye, as shown in Fig.1 (a). We can observe the vortex of typhoon because the wind blows the clouds to align with the local velocity field. However, curl does not always correspond to apparent vortex, as shown by the vector field in Fig.1 (b), which also represents a curled vector field.

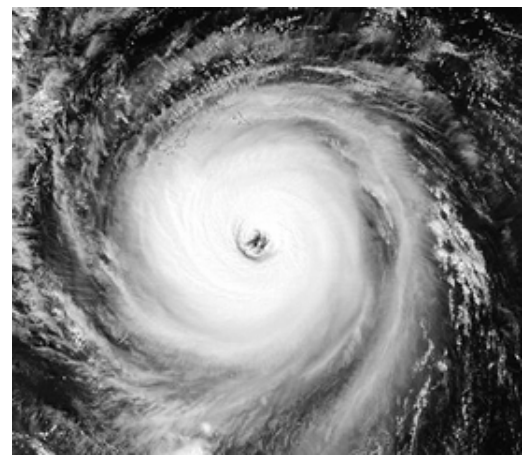

(a)

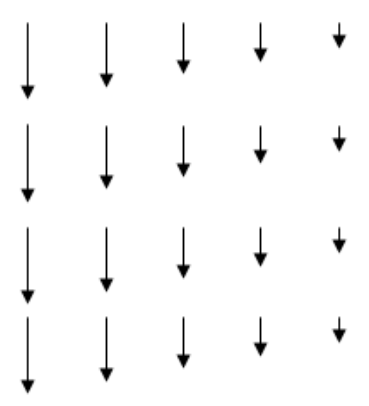

(b)

Fig. 1 (a) The vortex pattern of typhoon. (b) A curled vector field with no apparent vortex.

\section{Summary}

We have rigorously proved that the curl of a vector field is a pseudovector, by using differential chain rules for the transformed coordinates of the vector field under arbitrary space rotation. This kind of pseudovectors has important applications in physics, such as the angular velocity and the magnetic field [3]. We leave these applications to be discussed in the next paper.

\section{Acknowledgement}

This work was supported by the Teaching Enhancement Program of Zhejiang Ocean University and NSFC Grant No. 11505154, 11305141, 11104248, and 11204271.

\section{References}

[1]. Xiao-Ping Qin, Peng Li, et al, submitted to The 2nd International Conference on Mechanics, Materials and Structural Engineering (ICMMSE 2017). 
[2]. Information on: https://en.wikipedia.org/wiki/Curl_(mathematics)

[3]. J. D. Jackson, Classical Electrodynamics, John Wiley \& Sons, 1999. 